\title{
Structural phase transitions and phase ordering at surfaces probed by ultrafast LEED
}

\author{
Jan Gerrit Horstmann ${ }^{1,}$, Gero Storeck ${ }^{1}$, Bareld Wit ${ }^{1}$, Theo Diekmann ${ }^{1}$, Dennis Epp ${ }^{1}$, Kai \\ Rossnagel $^{2}$, Sascha Schäfer ${ }^{1}$, Simon Vogelgesang ${ }^{1}$, and Claus Ropers ${ }^{1}$ \\ ${ }^{1}$ IV. Physical Institute, University of Göttingen, Friedrich-Hund-Platz 1, 37077 Göttingen, Germany \\ ${ }^{2}$ Institute for Experimental and Applied Physics, University of Kiel, 24098 Kiel, Germany
}

\begin{abstract}
We demonstrate the capability of ultrafast low-energy electron diffraction to resolve phase-ordering kinetics and structural phase transitions on their intrinsic time scales with ultimate surface sensitivity.
\end{abstract}

\section{Introduction}

Solid state surface systems are particularly attractive because of their strongly altered physical and chemical properties compared with the bulk [1]. The lower dimensionality and broken symmetry of a surface gives rise to a variety of phenomena associated with modified electronic, lattice and spin structures, which in many cases are correlated with each other. We have recently developed ultrafast low-energy electron diffraction (ULEED) to explore optically-induced structural dynamics in the time-domain, exploiting the superior surface sensitivity and momentum resolution of LEED to resolve structural phase transitions and phase-ordering processes at surfaces [2,3]. Here, we present the observation of phase-ordering mechanisms and phason dynamics in the incommensurate charge density wave (CDW) phase of $1 \mathrm{~T}-\mathrm{TaS}_{2}$, enabled by time-resolved spot-profile analysis [3]. Furthermore, we report on the first time-resolved LEED studies of the structural phase transition associated with the insulator-metal transition in atomic Indium chains on a $\mathrm{Si}(111)$ surface.

\section{Experiment}

To realize ultrafast low-energy electron diffraction (ULEED), we employ low-energy electron pulses in a laser-pump/electron-probe scheme (Fig. 1a). Within this approach, a nanoscopic needle emitter triggered by $400 \mathrm{~nm}$ laser pulses (40 fs duration) via two-photon photoemission (2PPE) is utilized in a miniaturized electrostatic lens geometry as a highbrightness source. The resulting electron pulses exhibit a minimal duration down to $1.3 \mathrm{ps}$ at the sample for electron energies of $20-200 \mathrm{eV}[3,4]$. The strongly confined emission area leads to a high transversal coherence length of the pulsed electron beam, resulting in a momentum resolution of $\Delta \mathrm{k}_{\mathrm{s}}=0.03 \AA^{-1}$, which corresponds to a transfer width of $2 \pi / \Delta \mathrm{k}_{\mathrm{s}}=$ $21 \mathrm{~nm}$, at a spot size on the sample below $100 \mu \mathrm{m}$ (FWHM).

* Corresponding author: jan-gerrit.horstmann@uni-goettingen.de 

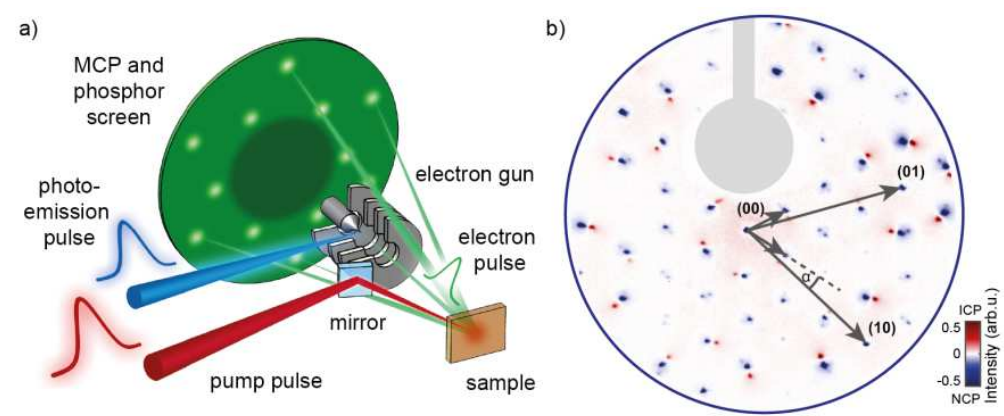

Fig. 1. a, Sketch of the optical-pump electron-probe scheme of the ULEED experiment. b, Difference image of the NC (blue) and IC (red) diffraction patterns (adapted from Ref. [3]).

\section{Results and Discussion}

We investigate the structural dynamics induced by the transformation of charge density waves at the surface of the quasi two-dimensional material $1 \mathrm{~T}-\mathrm{TaS}_{2}$ [3]. Specifically, we map the laser-driven transition from the room-temperature nearly-commensurate (NC) to the high temperature incommensurate (IC) CDW phase (Fig. 1b). Utilizing the high momentum resolution of the ULEED setup, we perform a thorough spot profile analysis of the appearing IC CDW diffraction peaks and observe a coarsening behaviour in the newly created IC phase (Fig. 1c). This growth of the IC CDW coherence length is attributed to the annihilation of dislocation-type topological defects. To corroborate our experimental observations, we perform numerical simulations of the IC CDW phase-ordering process in a time-dependent Ginzburg-Landau approach.

In a second set of experiments, we study collective phase-excitations, so-called phasons, in the IC phase [5]. After exciting the sample with an intense light pulse, we monitor the subsequent energy redistribution from the electron- into the phason-/phonon-subsystems. To this end, we determine the time-dependent temperature changes in the respective subsystems by a comparison of the pump-induced intensity suppressions of Bragg- and CDW-satellite-reflections. Our results indicate a strong initial energy transfer to the phason system, followed by slower phason-phonon equilibration, which suggests an inhibited electron-phason relaxation channel. We attribute this observation to a gap-induced decoupling of electron and phason systems, resulting in the pronounced generation of hot phasons.
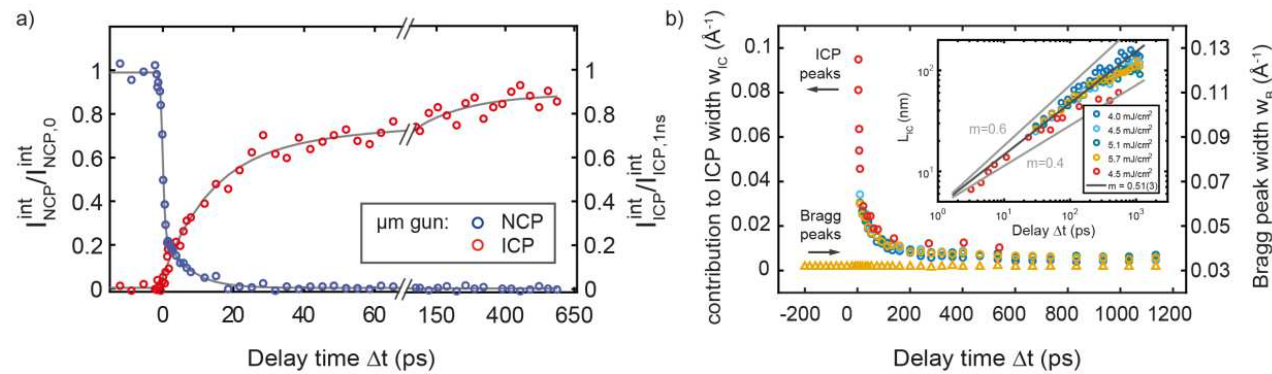

Fig. 2. a, Integrated CDW diffraction peak intensities recorded using the $\mu \mathrm{m}$-sized gun. b, Timedependent contribution to the width (FWHM) of the ICP diffraction peaks and width of lattice diffraction peaks for a range of optical-pump fluences. Red circles: data recorded with $\mu \mathrm{m}$-sized gun. Inset: A double-logarithmic plot of the correlation length corrected for the instrument response function, indicating a power-law scaling for the phase-ordering kinetics (adapted from Ref. [3]). 


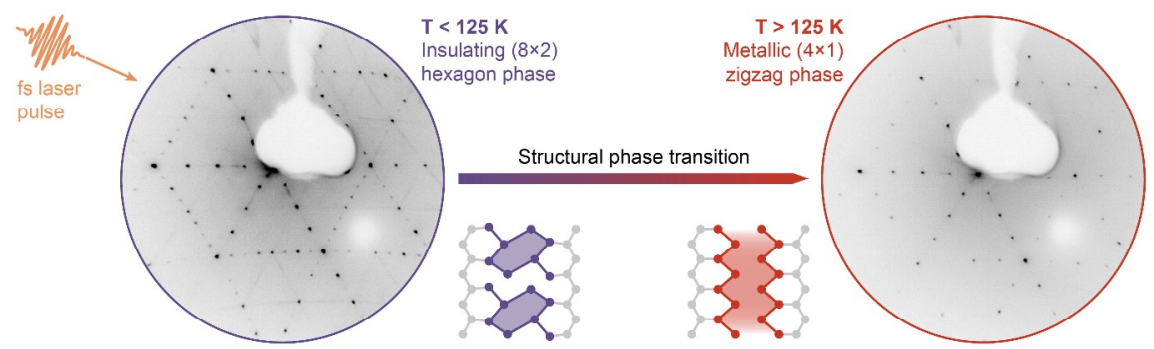

Fig. 3. Diffraction images and structure models of the low-temperature $(8 \times 2)$ and the hightemperature $(4 \times 1)$ phase of Indium atomic wires on a $\mathrm{Si}(111)$ surface recorded with $130 \mathrm{eV}$ electron pulses in our ULEED setup. The twofold streaks in the diffraction pattern of the $(8 \times 2)$ phase are the result of the missing correlation between adjacent atomic rows.

We further use the high surface sensitivity of our ULEED setup to study the surfacespecific transition between two CDW states of self-assembled Indium wires on Silicon. The sample is prepared by in situ evaporation of about one monolayer of Indium on a clean $\mathrm{Si}(111)$ surface followed by subsequent annealing and cooling down to $60 \mathrm{~K}$. Following optical excitation, the system undergoes a Peierls-type transition from a low-symmetry insulating to a high-symmetry metallic phase, accompanied by a structural phase transition between an $(8 \times 2)$ and a $(4 \times 1)$ superstructure [6]. We report on the first findings regarding the underlying structural dynamics probed by Ultrafast LEED.

\section{Summary and Conclusion}

In conclusion, using the example of $1 \mathrm{~T}-\mathrm{TaS}_{2}$, we reported the first systematic observation of ultrafast phase-ordering kinetics and phason dynamics enabled by the development of ultrafast low-energy electron diffraction (ULEED). We further explore the potential of this new technique by studying surface-specific structural phase transitions, such as Indium atomic chains on $\mathrm{Si}(111)$. Serving as an ideal complement to ultrafast angle-resolved photoelectron spectroscopy (ARPES), we believe that ULEED will pave the way for a greater understanding of a multitude of surface phenomena and consequently the optical control of complex surface dynamics, including phase transitions and chemical reactions.

\section{References}

1. J. M. Kosterlitz, D. J. Thouless, J. Phys. C 6, 1181 (1973)

2. M. Gulde, S. Schweda, G. Storeck, M. Maiti, H. K. Yu, A. M. Wodtke, S. Schäfer and C. Ropers, Science 345, 200 (2014)

3. S. Vogelgesang, G. Storeck, J. G. Horstmann, T. Diekmann, M. Sivis, S. Schramm, K. Rossnagel, S. Schäfer and C. Ropers, Nat. Phys. 14, 184 (2018)

4. G. Storeck, S. Vogelgesang, M. Sivis, S. Schäfer and C. Ropers, Struct. Dyn. 4, 044024 (2017)

5. A. W. Overhauser, Phys. Rev. B 3, 3173 (1971)

6. T. Frigge, B. Hafke, T. Witte, B. Krenzer, C. Streubühr, A. Samad Syed, V. Mikšić Trontl, I. Avigo, P. Zhou, M. Ligges, D. von der Linde, U. Bovensiepen, M. Horn-von Hoegen, S. Wippermann, A. Lücke, S. Sanna, U. Gerstmann and W. G. Schmidt, Nature 544, 207 (2017). 